\title{
Investigation of Monte Carlo uncertainties on Higgs boson searches using jet substructure
}

\author{
Peter Richardson ${ }^{\mathrm{a}}$, David Winn ${ }^{\mathrm{b}}$ \\ Institute of Particle Physics Phenomenology, Department of Physics, University of Durham, Durham DH1 3LE, UK
}

Received: 30 July 2012 / Revised: 20 September 2012 / Published online: 17 October 2012

(C) The Author(s) 2012. This article is published with open access at Springerlink.com

\begin{abstract}
We present an investigation of the dependence of searches for boosted Higgs bosons using jet substructure on the perturbative and non-perturbative parameters of the Herwig++ Monte Carlo event generator. Values are presented for a new tune of the parameters of the event generator, together with the an estimate of the uncertainties based on varying the parameters around the best-fit values.
\end{abstract}

\section{Introduction}

Monte Carlo simulations are an essential tool in the analysis of modern collider experiments. These event generators contain a large number of both perturbative and nonperturbative parameters which are tuned to a wide range of experimental data. While significant effort has been devoted to the tuning of the parameters to produce a best fit there has been much less effort understanding the uncertainties in these results. Historically a best fit result, or at best a small number of tunes, are produced and used to predict observables making it difficult to assess the uncertainty on any prediction. The "Perugia" tunes [1, 2] have addressed this by producing a range of tunes by varying specific parameters in the Pythia [3] event generator to produce an uncertainty.

Here we make use of the Professor Monte Carlo tuning system [4] to give an assessment of the uncertainty by varying all the parameters simultaneously about the best-fit values by diagonalizing the error matrix. This then allows us to systematically estimate the uncertainty on any Monte Carlo prediction from the tuning of the event generator. We will illustrate this by considering the uncertainty on jet substructure searches for the Higgs boson at the LHC.

As the LHC takes increasing amounts of data the discovery of the Higgs boson is likely in the near future. Once we

\footnotetext{
a e-mail: peter.richardson@durham.ac.uk

be-mail: d.e.winn@durham.ac.uk
}

have discovered the Higgs boson, most likely in the diphoton channel, it will be vital to explore other channels and determine if the properties of the observed Higgs boson are consistent with the Standard Model. For many years it was believed that it would be difficult, if not impossible, to observe the dominant $h^{0} \rightarrow b \bar{b}$ decay mode of a light Higgs boson. However, in recent years the use of jet substructure [5-20] offers the possibility of observing this mode. Jet substructure for $h^{0} \rightarrow b \bar{b}$ as a Higgs boson search channel, was first studied in Ref. [5] building on previous work of a heavy Higgs boson decaying to $W^{ \pm}$bosons [16], highenergy $W W$ scattering [21] and SUSY decay chains [22], and subsequently reexamined in Refs. [8, 15]. Recent studies at the LHC [23-25] have also shown this approach to be promising.

The study in Ref. [5] was carried out using the (FORTRAN) HERWIG 6.510 event generator [26, 27] together with the simulation of the underlying event using JIMMY 4.31 [28]. In order to allow the inclusion of new theoretical developments and improvements in non-perturbative modelling a new simulation based on the same physics philosophy Herwig++, currently version 2.6 [29, 30], is now preferred for the simulation of hadron-hadron collisions.

Herwig++ includes both an improved theoretical description of perturbative QCD radiation, in particular for radiation from heavy quarks, such as bottom, together with improved non-perturbative modeling, especially of multiple parton-parton scattering and the underlying event. In FORTRAN HERWIG a crude implementation of the dead-cone effect [31] meant that there was no radiation from heavy quarks for evolution scales below the quark mass, rather than a smooth suppression of soft collinear radiation. In Herwig++ an improved choice of evolution variable [32] allows evolution down to zero transverse momentum for radiation from heavy particles and reproduces the correct soft limit. There have also been significant developments of the multiple-parton scattering model of the underlying 
event [33, 34], including colour reconnections [35] and tuning to LHC data [36].

The background to jet substructure searches for the Higgs boson comes from QCD jets which mimic the decay of a boosted heavy particle. Although Herwig++ has performed well in some early studies of jet substructure [25, 37, 38], it is important that we understand the uncertainties in our modelling of the background jets which lie at the tail of the jet mass distribution.

In addition we improve the simulation of Higgs boson decay by implementing the next-to-leading-order (NLO) corrections to Higgs boson decay to heavy quarks in the POWHEG [39, 40] formalism.

In the next section we present our approach for the tuning of the parameters, which effect QCD radiation and hadronization, in Herwig++ together with the results of our new tune. We then recap the key features of the Butterworth, Davison, Rubin and Salam (BDRS) jet substructure technique of Ref. [5]. This is followed by our results using both the leading and next-to-leading-order matrix elements in Herwig++ with implementation of the next-to-leading-order Higgs boson decays and our estimate on the uncertainties.

\section{Tuning Herwig++}

Any jet substructure analysis is sensitive to changes in the simulation of initial- and final-state radiation, and hadronization. In particular the non-perturbative nature of the phenomenological hadronization model means there are a number of parameters which are tuned to experimental results. Herwig++ uses an improved angular-ordered parton shower algorithm $[29,32]$ to describe perturbative QCD radiation together with a cluster hadronization model [29, 41].

The Herwig++ cluster model is based on the concept of preconfinement [42]. At the end of the parton-shower evolution all gluons are non-perturbatively split into quarkantiquark pairs. All the partons can then be formed into colour-singlet clusters which are assumed to be hadron precursors and decay according to phase space into the observed hadrons. There is a small fraction of heavy clusters for which this is not a reasonable approximation which are therefore first fissioned into lighter clusters. The main advantage of this model, when coupled with the angularordered parton shower is that it has fewer parameters than the string model as implemented in the PYTHIA [3] event generator yet still gives a reasonable description of collider observables [43].

To tune Herwig++, and investigate the dependency of observables on the shower and hadronization parameters, the Professor Monte Carlo tuning system [4] was used. Professor uses the Rivet analysis framework [58] and a number of simulated event samples, with different Monte Carlo parameters, to parameterise the dependence of each observable ${ }^{1}$ used in the tuning on the parameters of the Monte Carlo event generator. A heuristic chi-squared function

$\chi^{\prime 2}(\boldsymbol{p})=\sum_{\mathcal{O}} w_{\mathcal{O}} \sum_{b \in \mathcal{O}} \frac{\left(f^{b}(\boldsymbol{p})-\mathcal{R}_{b}\right)^{2}}{\Delta_{b}^{2}}$,

is constructed where $\boldsymbol{p}$ is the set of parameters being tuned, $\mathcal{O}$ are the observables used each with weight $w_{\mathcal{O}}, b$ are the different bins in each observable distribution with associated experimental measurement $\mathcal{R}_{b}$, error $\Delta_{b}$ and Monte Carlo prediction $f^{b}(\boldsymbol{p})$. Weighting of those observables for which a good description of the experimental result is important is used in most cases. The parameterisation of the event generator response, $f(\boldsymbol{p})$, is then used to minimize the $\chi^{\prime 2}$ and find the optimum parameter values.

There are ten main free parameters which affect the shower and hadronization in Herwig++. These are shown in Table 1 along with their default values and allowed ranges.

The gluon mass, GluonMass, is required to allow the non-perturbative decay of gluons into $q \bar{q}$ pairs and controls the energy release in this process. PSplitLight, ClPowLight and ClMaxLight control the mass distributions of the clusters produced during the fission of heavy clusters. ClSmrLight controls the smearing of the direction of hadrons containing a (anti)quark from the perturbative evolution about the direction of the (anti)quark. AlphaMZ is strong coupling at the $Z^{0}$ boson mass and controls the amount of QCD radiation in the parton shower, while Qmin controls the infrared behaviour of the strong coupling. pTmin is the minimum allowed transverse momentum in the parton shower and controls the amount of radiation and the scale at which the perturbative evolution terminates. PwtDIquark and PwtSquark are the probabilities of selecting a diquark-antidiquark or $s \bar{s}$ quark pair from the vacuum during cluster splitting, and affect the production of baryons and strange hadrons respectively.

Previous experience of tuning Herwig++ has found that Qmin, GluonMass, ClSmrLight and ClPowLight to be flat, and so it was chosen to fix these at their default values [29].

To determine the allowed variation of these parameters Professor was used to tune the variables in Table 1 to the observables and weights found in Appendix A in Tables 5, 6,7 and 8 . The dependence of $\chi^{\prime 2}$ on the various parameters, about the minimum $\chi^{\prime 2}$ value, is then diagonalized.

The variation of the parameters along the eigenvectors in parameter space obtained corresponding to a certain change, $\Delta \chi^{\prime 2}$, in $\chi^{\prime 2}$ can then be used to predict the uncertainty in the Monte Carlo predictions for specific observables.

\footnotetext{
${ }^{1}$ Normally this is either an observation such as a multiplicity or a bin in a measured distribution.
} 
Table 1 The ten parameters to which the jet substructure is most sensitive with their default values, the allowed range of these values in Herwig++, the range scanned over and the new optimum value found from minimizing $\chi^{\prime 2}$

\begin{tabular}{lllll}
\hline Parameter & Default value & Allowed range & Scanned range & Optimum value \\
\hline Qmin & 0.935 & $\geq 0$ & $0.500-2.500$ & Fixed at default \\
GluonMass & 0.95 & $0-1$ & $0.75-1.00$ & Fixed at default \\
ClSmrLight & 0.78 & $0-2$ & $0.30-3.00$ & Fixed at default \\
ClPowLight & 1.28 & $0-10$ & $0.50-4.00$ & Fixed at default \\
pTmin & 1.00 & $\geq 0$ & $0.50-1.50$ & 0.88 \\
AlphaMz & 0.12 & $\geq 0$ & $0.10-0.12$ & 0.11 \\
ClMaxLight & 3.25 & $0-10$ & $3.00-4.20$ & 3.60 \\
PSplitLight & 1.20 & $0-10$ & $1.00-2.00$ & 0.90 \\
PwtDIquark & 0.49 & $0-10$ & $0.10-0.50$ & 0.33 \\
PwtSquark & 0.68 & $0-10$ & $0.50-0.80$ & 0.64 \\
\hline
\end{tabular}

In theory, if the $\chi^{\prime 2}$ measure for the parameterised generator response is actually distributed as a true $\chi^{2}$, then a change in the goodness of fit of one will correspond to a one sigma deviation from the minima, i.e. the best tune. In practice, even the best tune does not fit the data ideally and nor is the $\chi^{\prime 2}$ measure actually distributed according to a true $\chi^{2}$ distribution. This means that one cannot just use Professor to vary the parameters about the minima to a given deviation in the $\chi^{\prime 2}$ measure without using some subjective opinion on the quality of the results.

We simulated one thousand event samples with different randomly selected values of the parameters we were tuning. Six hundred of these were used to interpolate the generator response. All the event samples were used to select two hundred samples randomly two hundred times in order to assess the systematics of the interpolation and tuning procedure. A cubic interpolation of the generator response was used as this has been shown to give a good description of the Monte Carlo behaviour in the region of best generator response [4]. The parameters were varied between values shown in Table 1 . The quality of the interpolation was checked by comparing the $\chi^{\prime 2} / N_{\mathrm{df}}$, where $N_{\mathrm{df}}$ is the number of observable bins used in the tune, in the allowed parameter range on a parameter by parameter basis for the observables by comparing the interpolation response with actual generator response at the simulated parameter values. Bad regions were removed and the interpolation repeated leaving a volume in the 5-dimensional parameter space where the interpolation worked well.

Figure 1 shows the $\chi^{\prime 2} / N_{d f}$ distributions for two hundred tunes based on two hundred randomly selected event samples points for the cubic interpolation. The spread of these values gives an idea of the systematics of the tuning process showing that we have obtained a good fit for our parameterisation of the generator response.

The line indicates the tune which is based on a cubic interpolation from six hundred event samples. It is this interpolation which was used to vary $\chi^{\prime 2}$ about the minimum to assess the uncertainty on the measured distributions. During the tune it was discovered that PSplitLight was relatively insensitive to the observables used in the tune. As such, PSplitLight was fixed at the default value of 1.20 during the tune and subsequent $\chi^{\prime 2}$ variation.

Professor was used to vary $\chi^{\prime 2}$ about the minimum value, as described above, determining the allowed range for the parameters. As five parameters were eventually varied, there are 10 new sample points - one for each of the parameters and one "+" and one "-" along each eigenvector direction in parameter space.

We follow the example set by the parton distribution function (PDF) fitting groups in determining how much to allow $\chi^{\prime 2}$ to vary. Our situation is different to the PDF fitters in that we are using leading-order calculations with leading$\log$ accuracy in the parton shower, where they fit to next-toleading order calculations which gives better overall agreement with the observables used. Generally, PDF groups fit to fully inclusive variables, where as we have fitted to more exclusive processes and by nature, these are more model dependent, in particular hadronization.

In Refs. [45, 46] these issues are explored in terms of PDFs and the allowed variation is related to a tolerance parameter $T$, where

$\Delta \chi_{\text {global }}^{\prime 2} \leq T^{2}$.

A tolerance parameter of $T \approx 10$ to 15 is generally chosen for the PDF groups, where they are fitting to around 1300 data points. As our fit is likley to have a higher $\chi^{2}$ than their fit due to the aforementioned reasons, and that we fit to a greater number of parameters, we will have a higher tolerance parameter.

In our fit, we have 1665 degrees-of-freedom and we examined various changes in $\chi^{\prime 2}$, whilst considering the effects of the precision data from LEP. A variation of $\Delta \chi^{\prime 2} / N_{\mathrm{df}}=5$, equivalent to $T \approx 90$, seems, subjectively to keep the LEP data within reasonable limits while a variation of $\Delta \chi^{\prime 2} / N_{\mathrm{df}}=10$, i.e. $T \approx 130$ is too large. Anything less $T \approx 40$ had very little variation and was therefore 


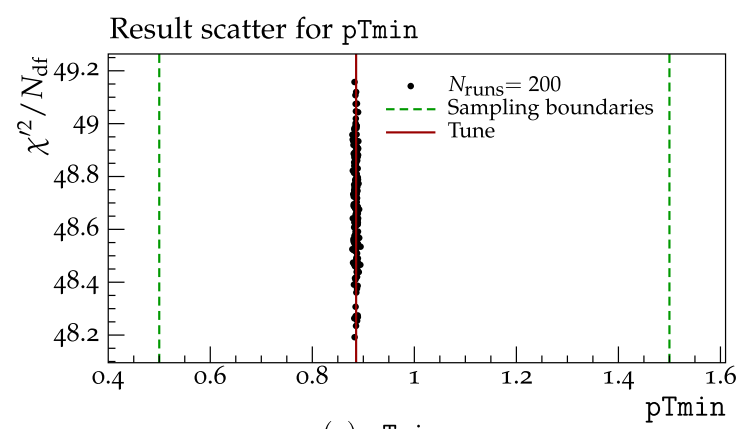

(a) pTmin

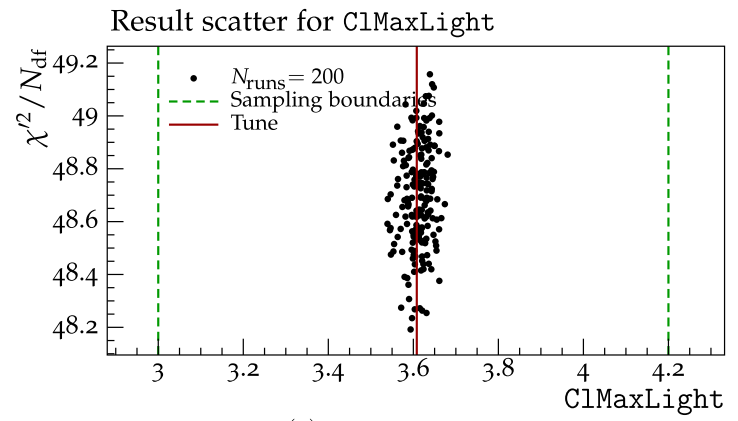

(c) ClMaxLight

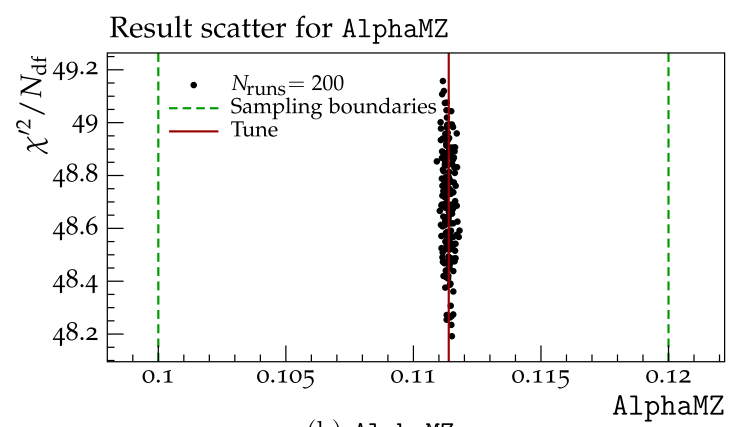

(b) AlphaMZ

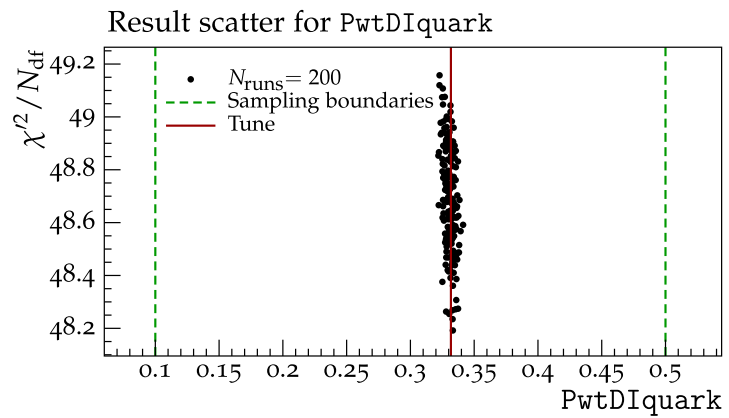

(d) PwtDIquark

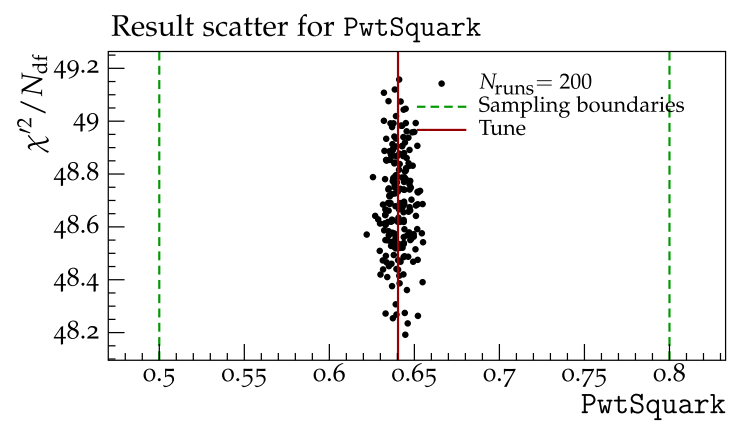

(e) PwtSquark

Fig. 1 The $\chi^{\prime 2} / N_{\mathrm{df}}$ distributions for the parameters that were varied from their default values whilst determining the error tune. The scatter of the results gives a representation of the systematics of tuning procedure

Table 2 The five directions corresponding to the error tune for a $\Delta \chi^{\prime 2} / N_{\mathrm{df}}=5$ and the values the parameters take in each direction

\begin{tabular}{|c|c|c|c|c|c|c|c|c|c|c|}
\hline \multirow[t]{3}{*}{ Parameter } & \multicolumn{10}{|c|}{ Direction } \\
\hline & \multicolumn{2}{|l|}{1} & \multicolumn{2}{|l|}{2} & \multicolumn{2}{|l|}{3} & \multicolumn{2}{|l|}{4} & \multicolumn{2}{|l|}{5} \\
\hline & + & - & + & - & + & - & + & - & + & - \\
\hline pTmin & 0.88 & 0.88 & 0.88 & 0.88 & 0.84 & 0.93 & 0.87 & 0.90 & 0.89 & 0.87 \\
\hline AlphaMz & 0.11 & 0.11 & 0.10 & 0.12 & 0.12 & 0.11 & 0.12 & 0.11 & 0.12 & 0.11 \\
\hline ClMaxLight & 3.61 & 3.61 & 3.61 & 3.61 & 3.60 & 3.62 & 3.66 & 3.55 & 3.54 & 3.67 \\
\hline PwtDIquark & 0.46 & 0.23 & 0.33 & 0.33 & 0.33 & 0.33 & 0.33 & 0.33 & 0.33 & 0.33 \\
\hline PwtSquark & 0.64 & 0.64 & 0.64 & 0.64 & 0.64 & 0.64 & 0.62 & 0.67 & 0.51 & 0.78 \\
\hline
\end{tabular}

deemed inappropriate. The values for both $\Delta \chi^{\prime 2} / N_{\mathrm{df}}=5$ and $\Delta \chi^{\prime 2} / N_{\mathrm{df}}=10$ are shown in are shown in Tables 2 and 3 respectively.

The Professor tune was then compared with the internal Herwig++ tuning procedure [29] as not all analyses that are in the internal Herwig++ tuning system are available in Rivet and subsequently accessible to Professor. Looking at Fig. 4 it is found that PSplitLight at a value of 0.90 is favoured and gives a significant reduction in the $\chi^{\prime 2} / N_{\mathrm{df}}$. It was therefore decided to use the values obtained from minimisation procedure, but using the value of 0.90 for PSplitLight to maintain a good overall description of the data. The new 
Table 3 The five directions corresponding to the error tune for a $\Delta \chi^{\prime 2} / N_{\mathrm{df}}=10$ and the values the parameters take in each direction

\begin{tabular}{|c|c|c|c|c|c|c|c|c|c|c|}
\hline \multirow[t]{3}{*}{ Parameter } & \multicolumn{10}{|c|}{ Direction } \\
\hline & \multicolumn{2}{|l|}{1} & \multicolumn{2}{|l|}{$\underline{2}$} & \multicolumn{2}{|l|}{3} & \multicolumn{2}{|l|}{4} & \multicolumn{2}{|l|}{5} \\
\hline & + & - & + & - & + & - & + & - & + & - \\
\hline pTmin & 0.88 & 0.88 & 0.88 & 0.88 & 0.82 & 0.95 & 0.86 & 0.90 & 0.89 & 0.87 \\
\hline AlphaMz & 0.11 & 0.11 & 0.10 & 0.12 & 0.12 & 0.10 & 0.12 & 0.10 & 0.12 & 0.11 \\
\hline ClMaxLight & 3.61 & 3.61 & 3.61 & 3.61 & 3.59 & 3.63 & 3.68 & 3.52 & 3.52 & 3.70 \\
\hline PwtDIquark & 0.51 & 0.19 & 0.33 & 0.33 & 0.33 & 0.33 & 0.33 & 0.33 & 0.33 & 0.33 \\
\hline PwtSquark & 0.64 & 0.64 & 0.64 & 0.64 & 0.65 & 0.64 & 0.61 & 0.68 & 0.46 & 0.84 \\
\hline
\end{tabular}

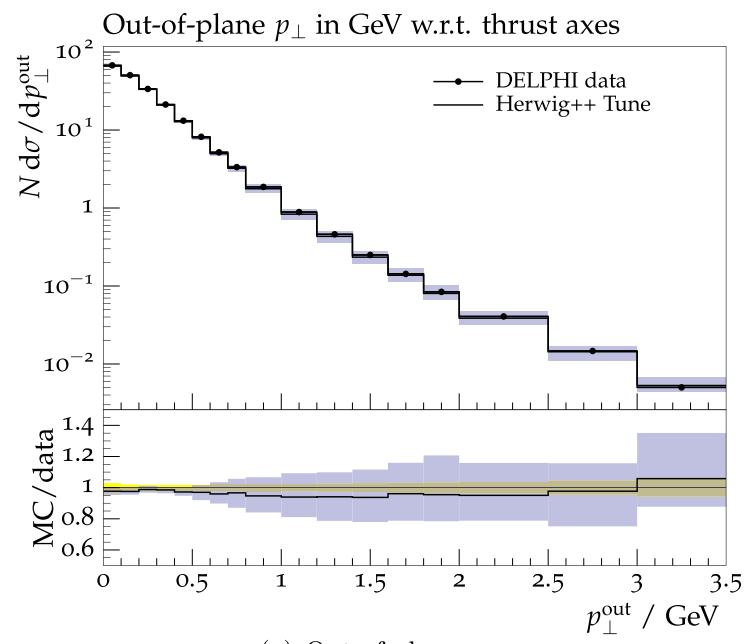

(a) Out-of-plane $p_{T}$

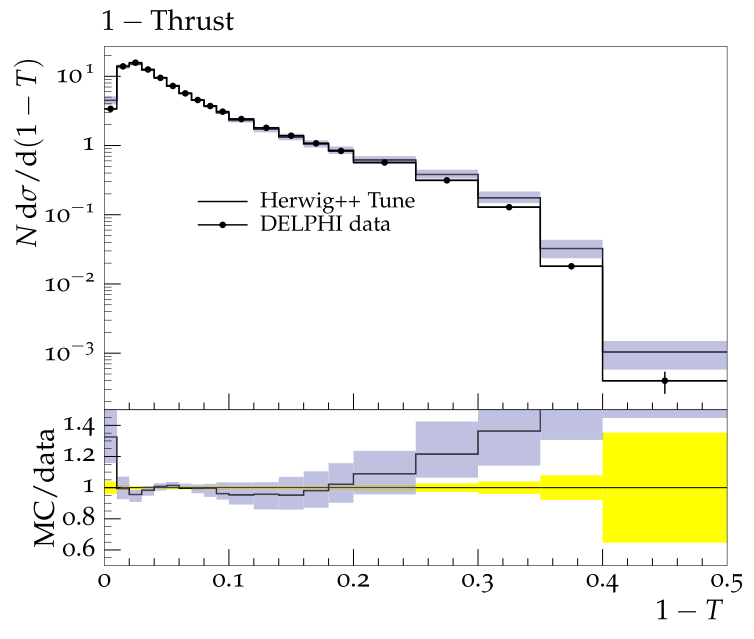

(b) 1-Thrust

Fig. 2 Results from the DELPHI [44] analysis of out-of-plane $p_{T}$ with-respect-to the thrust axis and 1-thrust showing the new tune and the envelopes corresponding to a change in $\Delta \chi^{\prime 2} / N_{\mathrm{df}}=5$

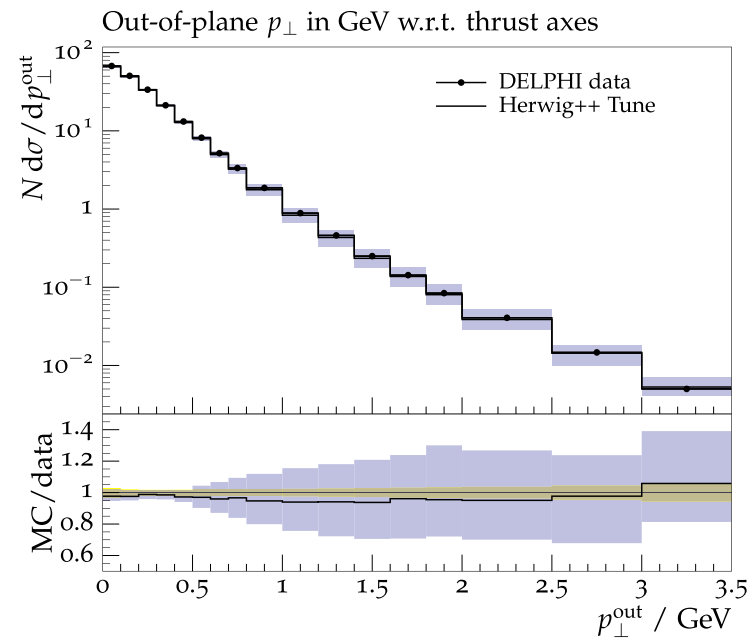

(a) Out-of-plane $p_{T}$

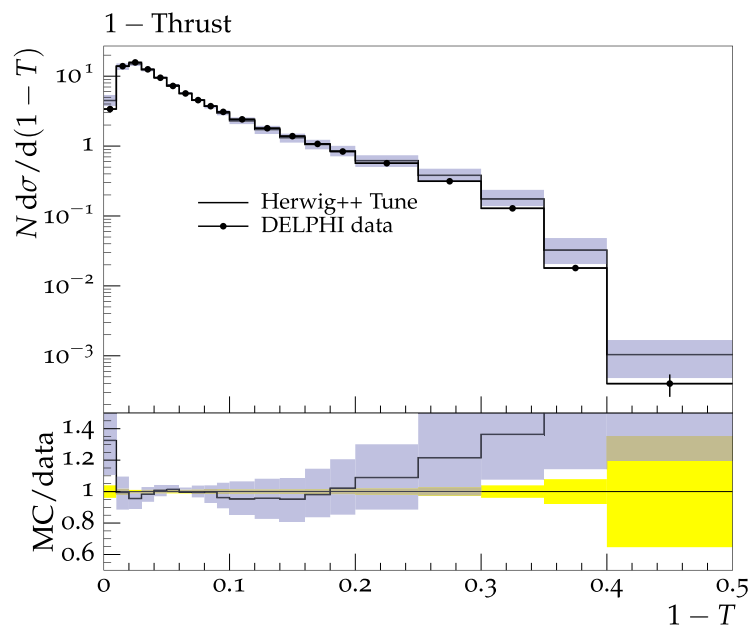

(b) 1-Thrust

Fig. 3 Results from the DELPHI [44] analysis of out of plane $p_{T}$ with-respect-to the thrust axis and 1-thrust showing the new tune and the envelopes corresponding to a change in $\Delta \chi^{\prime 2} / N_{\mathrm{df}}=10$

minima for the QCD parameters are summarized in the Table 1. Examples of the new tune and the uncertainty band are shown in Figs. 2 and 3 for the out-of-plane transverse momentum and thrust measured by DELPHI [44].
These error tune values can now be used to predict the uncertainty from the tuning of the shower parameters on any observable. In the next section we will present an example of using these tunes to estimate the uncertainty on the pre- 


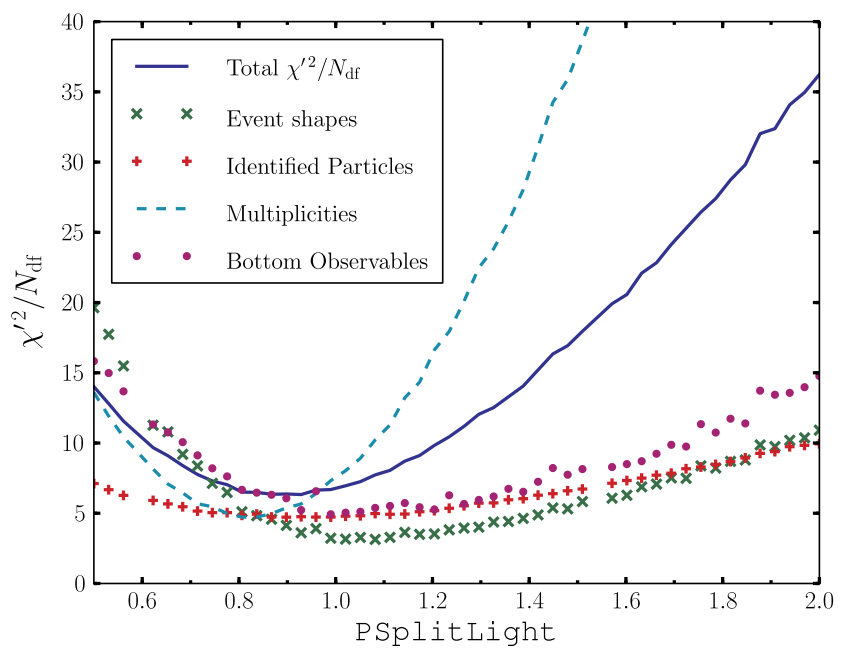

Fig. 4 A scan of PSplitLight using the internal Herwig++ tuning system with the other parameters fixed at their new tuned value. From the total $\chi^{\prime 2} / N_{\mathrm{df}}$ we see that a value of 0.90 for PSplitLight is favoured at the new tuned parameters driven by the multiplicities

dictions for searches for the Higgs boson using the BDRS jet substructure method.

\section{Jet substructure boosted Higgs}

The analysis of Ref. [5] uses a number of different channels for the production of the Higgs boson decaying to $b \bar{b}$ in association with an electroweak gauge boson, i.e. the production of $h^{0} Z^{0}$ and $h^{0} W^{ \pm}$. Reference [5] uses the fact that the Higgs boson predominantly decays to $b \bar{b}$ in a jet substructure analysis to extract the signal of a boosted Higgs boson above the various backgrounds. Their study found that the Cambridge-Aachen algorithm $[47,48]$ with radius parameter $R=1.2$ gave the best results when combined with their jet substructure technique. For our study, we used the Cambridge-Aachen algorithm as implemented in the FastJet package [49]. Three different event selection criteria are used:

(a) a lepton pair with $80 \mathrm{GeV}<m_{l^{+} l^{-}}<100 \mathrm{GeV}$ and $p_{T}>p_{T}^{\min }$ to select events for $Z^{0} \rightarrow \ell^{+} \ell^{-}$;

(b) missing transverse momentum $p_{T}>p_{T}^{\text {min }}$ to select events with $Z^{0} \rightarrow \nu \bar{v}$;

(c) missing transverse momentum $p_{T}>30 \mathrm{GeV}$ and a lepton with $p_{T}>30 \mathrm{GeV}$ consistent with the presence of a W boson with $p_{T}>p_{T}^{\min }$ to select events with $W \rightarrow \ell v$;

where $p_{T}^{\min }=200 \mathrm{GeV}$.

In addition the presence of a hard jet with $p_{T_{j}}>p_{T}^{\min }$ with substructure is required. The substructure analysis of Ref. [5] proceeds with the hard jet $j$ with some radius $R_{j}$, a mass $m_{j}$ and in a mass-drop algorithm:
1. the two subjets which were merged to form the jet, ordered such that the mass of the first jet $m_{j_{1}}$ is greater than that of the second jet $m_{j_{2}}$, are obtained;

2. if $m_{j_{1}}<\mu m_{j}$ and

$$
y=\frac{\min \left(p_{T j_{1}}^{2}, p_{T j_{2}}^{2}\right)}{m_{j}^{2}} \Delta R_{j_{1}, j_{2}}^{2}>y_{\text {cut }},
$$

where $\Delta R_{j_{1}, j_{2}}^{2}=\left(y_{j_{1}}-y_{j_{2}}\right)^{2}+\left(\phi_{j_{1}}-\phi_{j_{2}}\right)^{2}$, and $p_{T j_{1,2}}$, $\eta_{j_{1,2}}, \phi_{j_{1,2}}$ are the transverse momenta, rapidities and azimuthal angles of jets 1 and 2, respectively, then $j$ is in the heavy particle region. If the jet is not in the heavy particle region the procedure is repeated using the first jet.

This algorithm requires that $j_{1,2}$ are $b$-tagged and takes $\mu=0.67$ and $y_{\text {cut }}=0.09$. A uniform $b$-tagging efficiency of $60 \%$ was used with a uniform mistagging probability of $2 \%$. The heavy jet selected by this procedure is considered to be the Higgs boson candidate jet. Finally, there is a filtering procedure on the Higgs boson candidate jet, $j$. The jet, $j$, is resolved on a finer scale by setting a new radius $R_{\text {filt }}=\min \left(0.3, R_{b \bar{b}} / 2\right)$, where from the previous massdrop condition, $R_{b \bar{b}}=\Delta R_{j_{1}, j_{2}}^{2}$. The three hardest subjects of this filtering process are taken to be the Higgs boson decay products, where the two hardest are required to be $b$-tagged.

All three analyses require that:

- after the reconstruction of the vector boson, there are no additional leptons with pseudorapidity $|\eta|<2.5$ and $p_{T}>30 \mathrm{GeV}$;

- other than the Higgs boson candidate, there are no additional $b$-tagged jets with pseudorapidity $|\eta|<2.5$ and $p_{T}>50 \mathrm{GeV}$.

In addition, due to top contamination, criterion (c) requires that other than the Higgs boson candidate, there are no additional jets with $|\eta|<3$ and $p_{T}>30 \mathrm{GeV}$. For all events, the candidate Higgs boson jet should have $p_{T}>$ $p_{T}^{\min }$. The analyses were implemented using the Rivet system [58].

The plots shown in Fig. 5 use the leading-order matrix elements for the production and decay of Higgs boson but the $W, Z$ and top [50] have matrix element corrections for their decays. The plots shown in Fig. 6 have leading-order $t \bar{t}$ production, leading-order vector boson plus jet production (with the same matrix element corrections as the LO matrix elements) but the NLO vector boson pair production [51] and NLO vector and Higgs boson associated production [52]. In addition we have implemented the corrections to the decay $h^{0} \rightarrow b \bar{b}$ in the POWHEG scheme, as described in Appendix B. The signal significances are outlined in Table 4. 
Fig. 5 Results for the reconstructed Higgs boson mass distribution using leading-order matrix elements. A SM Higgs boson was assumed with a mass of $115 \mathrm{GeV}$. In addition to the full result the contribution from top quark pair production $(t \bar{t})$, the production of $W^{ \pm}(\mathrm{W}+\mathrm{Jet})$ and $Z^{0}(\mathrm{Z}+\mathrm{Jet})$ bosons in association with a hard jet, vector boson pair production (VV) and the production of a vector boson in association with the Higgs boson $(\mathrm{V}+$ Higgs $)$, are shown

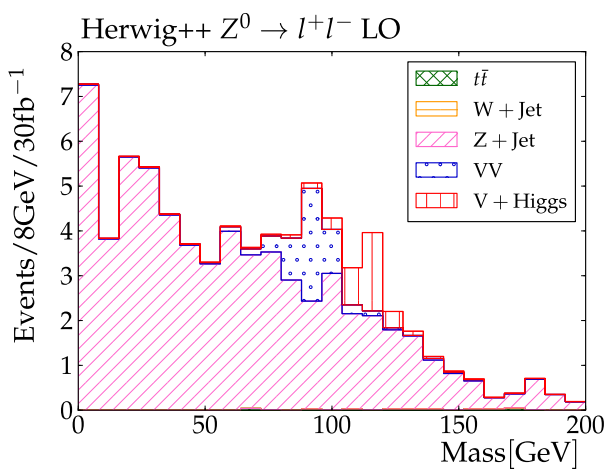

(a) Selection criterion (a)

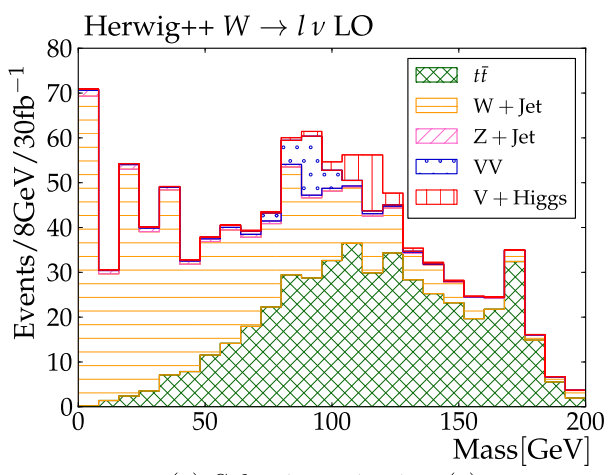

(c) Selection criterion (c)

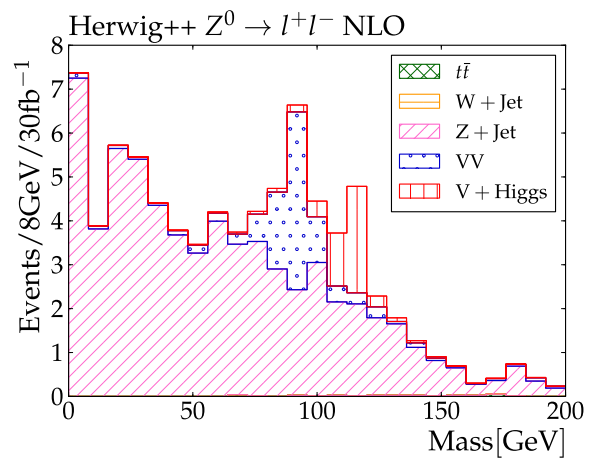

(a) Selection criterion (a)

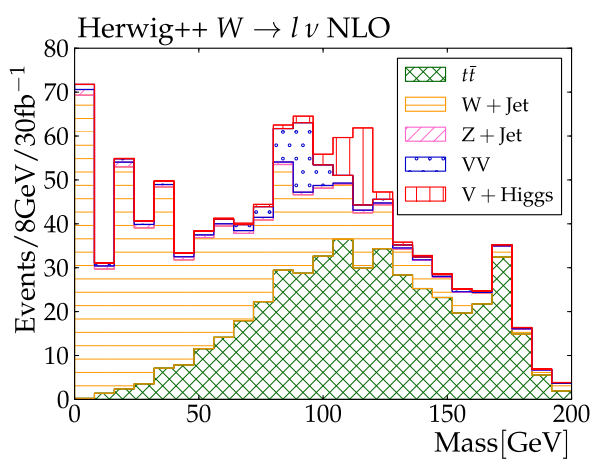

(c) Selection criterion (c)

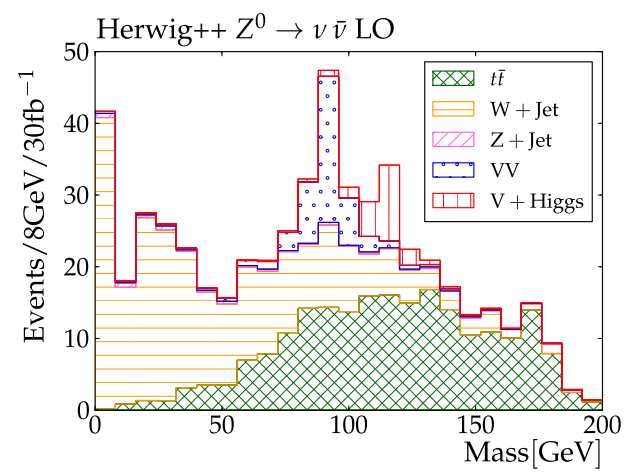

(b) Selection criterion (b)

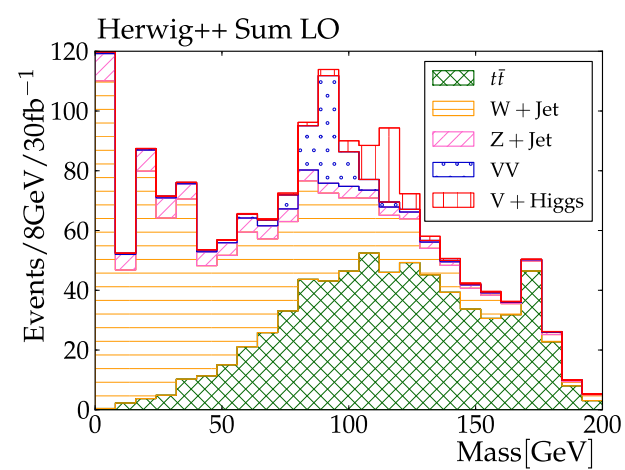

(d) Sum of criteria (a), (b) and (c)

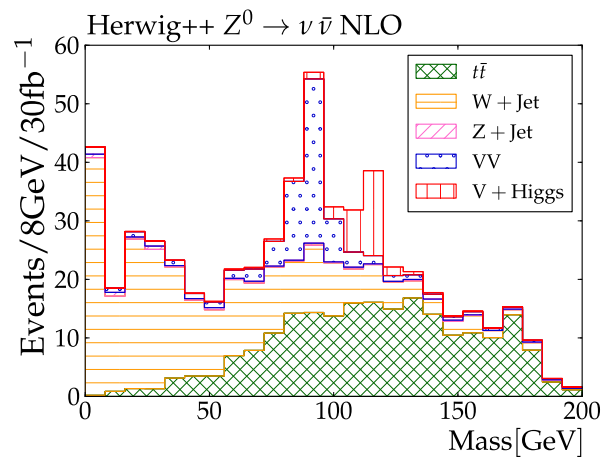

(b) Selection criterion (b)

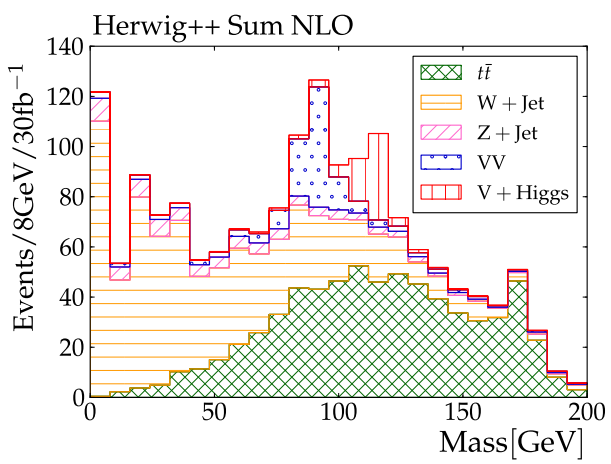

(d) Sum of criteria (a), (b) and (c)
The uncertainties due to the Monte Carlo simulation are shown as bands in Figs. 7 and 8. As there are correlations between the different processes the uncertainty is determined for the sum of all processes. Whilst it would be possible to show the envelope for the individual processes, this would not offer any information on the envelope for the sum of the 
Fig. 7 Results for the reconstructed Higgs boson mass distribution using leading-order matrix elements. A SM Higgs boson was assumed with a mass of $115 \mathrm{GeV}$. The envelope shows the uncertainty from the Monte Carlo simulation

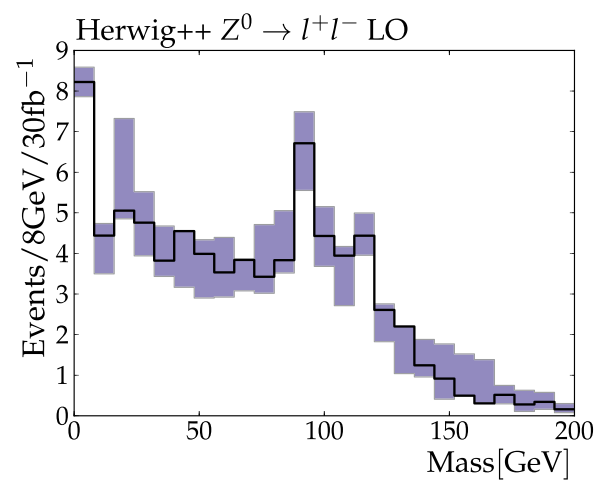

(a) Selection criterion (a)

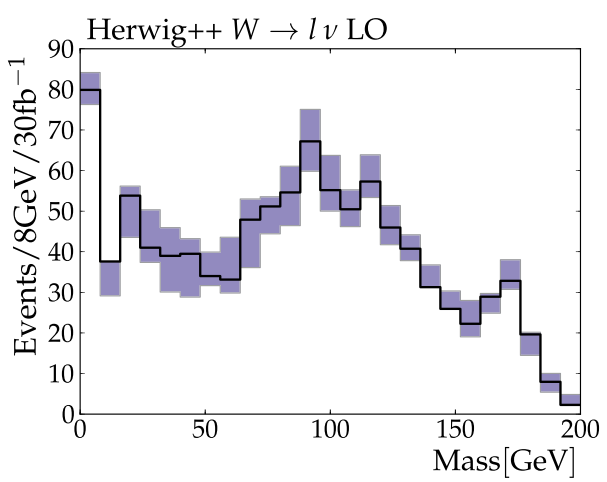

(c) Selection criterion (c)

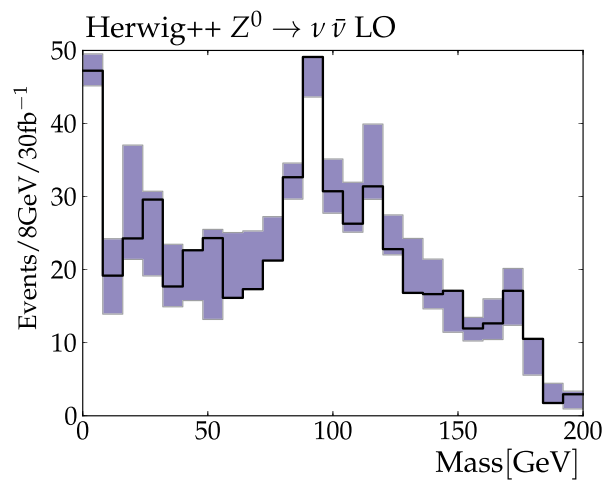

(b) Selection criterion (b)

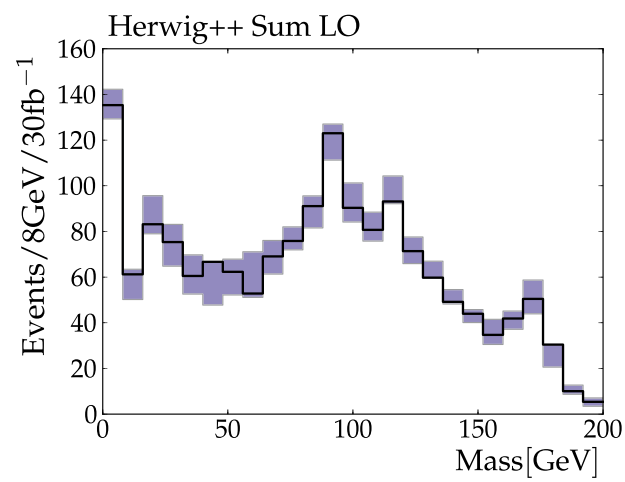

(d) Sum of criteria (a), (b) and (c)
Fig. 8 Results for the reconstructed Higgs boson mass distribution using leading-order matrix elements for top quark pair production, and the production of $W^{ \pm}$and $Z^{0}$ bosons in association with a hard jet. The next-to-leading-order corrections are included for vector boson pair production and the production of a vector boson in association with the Higgs boson as well as in the decay of the Higgs boson, $h^{0} \rightarrow b \bar{b}$. A SM Higgs boson was assumed with a mass of $115 \mathrm{GeV}$. The envelope shows the uncertainty from the Monte Carlo simulation

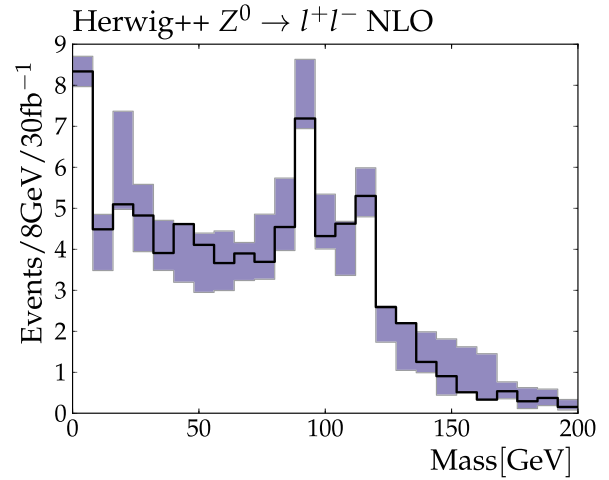

(a) Selection criterion (a)

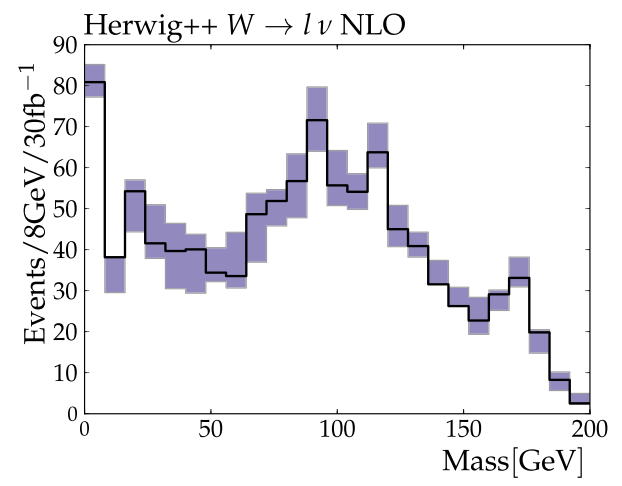

(c) Selection criterion (c)

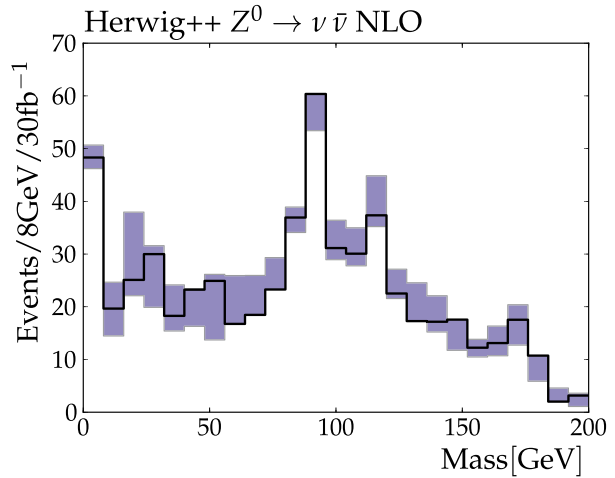

(b) Selection criterion (b)

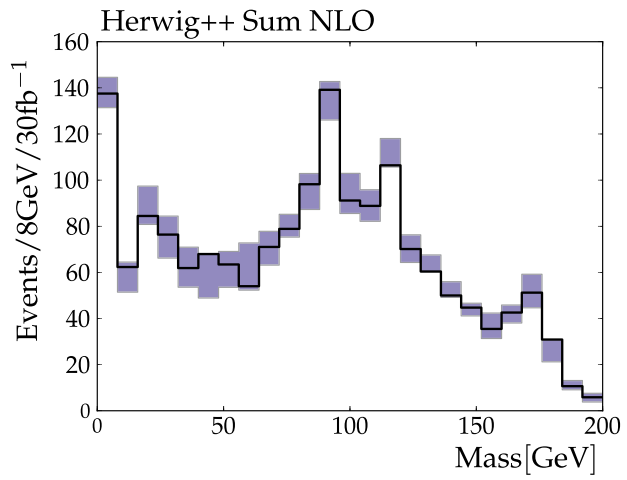

(d) Sum of criteria (a), (b) and (c) 
Table 4 The significance of the different processes for the leadingand next-to-leading-order matrix elements. The significance is calculated using all masses in the range $112-120 \mathrm{GeV}$

\begin{tabular}{|c|c|c|c|}
\hline \multicolumn{4}{|l|}{ Significance } \\
\hline Process & Order & $\begin{array}{l}\frac{S}{\sqrt{B}} \\
\text { Herwig++ default }\end{array}$ & $\begin{array}{l}\frac{S}{\sqrt{B}} \\
\text { Herwig++ tune }\end{array}$ \\
\hline \multirow[t]{2}{*}{$Z^{0} \rightarrow l^{+} l^{-}$} & LO & 1.17 & $1.24_{-0.11}^{+0.36}$ \\
\hline & NLO & 1.57 & $1.96_{-0.30}^{+0.29}$ \\
\hline \multirow[t]{2}{*}{$Z^{0} \rightarrow v \bar{v}$} & LO & 2.18 & $2.89_{-0.60}^{+0.19}$ \\
\hline & NLO & 2.95 & $4.04_{-0.90}^{+0.25}$ \\
\hline \multirow[t]{2}{*}{$W \rightarrow l v$} & LO & 1.88 & $2.32_{-0.27}^{+0.15}$ \\
\hline & NLO & 2.63 & $3.20_{-0.36}^{+0.29}$ \\
\hline \multirow[t]{2}{*}{ Total } & LO & 2.98 & $3.71_{-0.53}^{+0.29}$ \\
\hline & NLO & 4.09 & $5.20_{-0.81}^{+0.43}$ \\
\hline
\end{tabular}

processes which is the result of interest. In addition the uncertainty on the significance is shown in Table 4.

\section{Conclusions}

Monte Carlo simulations are an essential tool in the analysis of modern collider experiments. While significant effort has been devoted to the tuning of the parameters to produce a best fit there has been much less effort understanding the uncertainties in these results. In this paper we have produced a set of tunes which can be used to assess this uncertainty using the Herwig++ Monte Carlo event generator.

We then used these tunes to assess the uncertainties on the mass-drop analysis of Ref. [5] using Herwig++ with both leading- and next-to-leading-order matrix elements including a POWHEG simulation of the decay $h^{0} \rightarrow b \bar{b}$.

We find that while the jet substructure technique has significant potential as a Higgs boson discovery channel, we need to be confident of our tunes to investigate this with Monte Carlo simulations.

The error tunes and procedure here can now be used in other analyses where the uncertainty due to the Monte Carlo simulation is important.

Acknowledgements We are grateful to all the other members of the Herwig++ collaboration and Hendrik Hoeth for valuable discussions and the authors of Ref. [5] for help in reproducing their results. We acknowledge the use of the UK Grid for Particle Physics in producing the results. This work was supported by the Science and Technology Facilities Council. DW acknowledges support by the STFC studentship ST/F007299/1.

Open Access This article is distributed under the terms of the Creative Commons Attribution License which permits any use, distribu- tion, and reproduction in any medium, provided the original author(s) and the source are credited.

\section{Appendix A: Observables and weights used to tune Herwig++}

The weights and observables used in the Professor tuning system are outlined in Tables 5, 6, 7 and 8.

Table 5 Observables used in the tuning and associated weights for observables taken from [53]

\begin{tabular}{lllll}
\hline Observable & Weight & & Observable & Weight \\
\cline { 1 - 2 } \cline { 5 - 6 }$K^{* \pm}(892)$ spectrum & 1.0 & & $\Lambda^{0}$ spectrum & 1.0 \\
$\rho$ spectrum & 1.0 & & $\pi^{0}$ spectrum & 1.0 \\
$\omega(782)$ spectrum & 1.0 & & $p$ spectrum & 1.0 \\
$\Xi^{-}$spectrum & 1.0 & & $\eta^{\prime}$ spectrum & 1.0 \\
$K^{* 0}(892)$ spectrum & 1.0 & & $\Xi^{0}(1530)$ spectrum & 1.0 \\
$\phi$ spectrum & 1.0 & & $\pi^{ \pm}$spectrum & 1.0 \\
$\Sigma^{ \pm}(1385)$ spectrum & 1.0 & & $\eta$ spectrum & 1.0 \\
$\gamma$ spectrum & 1.0 & & $K^{0}$ spectrum & 1.0 \\
\cline { 5 - 6 }$K^{ \pm}$spectrum & 1.0 & &
\end{tabular}

Table 6 Observables used in the tuning and associated weights for observables taken from [44]

\begin{tabular}{ll}
\hline Observable & Weight \\
\hline Sphericity, $S$ & 1.0 \\
Energy-energy correlation, EEC & 1.0 \\
Aplanarity, $A$ & 2.0 \\
Mean out-of-plane $p_{\perp}$ in GeV w.r.t. thrust axes vs. $x_{p}$ & 1.0 \\
Mean charged multiplicity & 150.0 \\
Mean $p_{\perp}$ in GeV vs. $x_{p}$ & 1.0 \\
Planarity, $P$ & 1.0 \\
Thrust major, $M$ & 1.0 \\
Oblateness $=M-m$ & 1.0 \\
Out-of-plane $p_{\perp}$ in GeV w.r.t. sphericity axes & 1.0 \\
$D$ parameter & 1.0 \\
$1-$ Thrust & 1.0 \\
Out-of-plane $p_{\perp}$ in GeV w.r.t. thrust axes & 1.0 \\
Log of scaled momentum, log $\left(1 / x_{p}\right)$ & 1.0 \\
In-plane $p_{\perp}$ in GeV w.r.t. sphericity axes & 1.0 \\
In-plane $p_{\perp}$ in $\mathrm{GeV}$ w.r.t. thrust axes & 1.0 \\
Thrust minor, $m$ & 2.0 \\
$C$ parameter & 1.0 \\
Scaled momentum, $x_{p}=|p| /\left|p_{\text {beam }}\right|$ & 1.0 \\
\hline
\end{tabular}


Table 7 Multiplicities used in the tuning and associated weights for observables taken from [59]

\begin{tabular}{|c|c|c|c|}
\hline Observable & Weight & Observable & Weight \\
\hline Mean $\rho^{0}(770)$ multiplicity & 10.0 & Mean $\chi_{c 1}(3510)$ multiplicity & 10.0 \\
\hline Mean $\Delta^{++}(1232)$ multiplicity & 10.0 & Mean $D^{+}$multiplicity & 10.0 \\
\hline Mean $K^{*+}(892)$ multiplicity & 10.0 & Mean $\Sigma^{+}$multiplicity & 10.0 \\
\hline Mean $\Sigma^{0}$ multiplicity & 10.0 & Mean $f_{1}(1285)$ multiplicity & 10.0 \\
\hline Mean $\Lambda_{b}^{0}$ multiplicity & 10.0 & Mean $f_{2}(1270)$ multiplicity & 10.0 \\
\hline Mean $K^{+}$multiplicity & 10.0 & Mean $J / \psi(1 S)$ multiplicity & 10.0 \\
\hline Mean $\Xi^{0}(1530)$ multiplicity & 10.0 & Mean $B_{u}^{+}$multiplicity & 10.0 \\
\hline Mean $\Lambda(1520)$ multiplicity & 10.0 & Mean $B^{*} *$ multiplicity & 10.0 \\
\hline Mean $D_{s}^{*+}(2112)$ multiplicity & 10.0 & Mean $\Lambda_{c}^{+}$multiplicity & 10.0 \\
\hline Mean $\Sigma^{-}(1385)$ multiplicity & 10.0 & Mean $D^{0}$ multiplicity & 10.0 \\
\hline Mean $f_{1}(1420)$ multiplicity & 10.0 & Mean $f_{2}^{\prime}(1525)$ multiplicity & 10.0 \\
\hline Mean $\phi(1020)$ multiplicity & 10.0 & Mean $\Sigma^{ \pm}$multiplicity & 10.0 \\
\hline Mean $K_{2}^{* 0}(1430)$ multiplicity & 10.0 & Mean $D_{s 2}^{+}$multiplicity & 10.0 \\
\hline Mean $\Omega^{-}$multiplicity & 10.0 & Mean $K^{* 0}(892)$ multiplicity & 10.0 \\
\hline Mean $\Sigma^{ \pm}(1385)$ multiplicity & 10.0 & Mean $\Sigma^{-}$multiplicity & 10.0 \\
\hline Mean $\psi(2 S)$ multiplicity & 10.0 & Mean $\pi^{+}$multiplicity & 10.0 \\
\hline Mean $D^{*+}(2010)$ multiplicity & 10.0 & Mean $f_{0}(980)$ multiplicity & 10.0 \\
\hline Mean $B^{*}$ multiplicity & 10.0 & Mean $\Sigma^{+}(1385)$ multiplicity & 10.0 \\
\hline Mean $\pi^{0}$ multiplicity & 10.0 & Mean $D^{+} s$ multiplicity & 10.0 \\
\hline Mean $\eta$ multiplicity & 10.0 & Mean $p$ multiplicity & 10.0 \\
\hline Mean $a_{0}^{+}(980)$ multiplicity & 10.0 & Mean $B_{s}^{0}$ multiplicity & 10.0 \\
\hline Mean $D_{s 1}^{+}$multiplicity & 10.0 & Mean $K^{0}$ multiplicity & 10.0 \\
\hline Mean $\rho^{+}(770)$ multiplicity & 10.0 & Mean $B^{+}, B_{d}^{0}$ multiplicity & 10.0 \\
\hline Mean $\Xi^{-}$multiplicity & 10.0 & Mean $\Lambda$ multiplicity & 10.0 \\
\hline Mean $\omega$ (782) multiplicity & 10.0 & Mean $\eta^{\prime}(958)$ multiplicity & 10.0 \\
\hline Mean $\Upsilon(1 S)$ multiplicity & 10.0 & & \\
\hline
\end{tabular}

Table 8 Observables used in the tuning and associated weights for observables taken from [60]

\begin{tabular}{ll}
\hline Observable & Weight \\
\hline$b$ quark fragmentation function $f\left(x_{B}^{\text {weak }}\right)$ & 7.0 \\
Mean of $b$ quark fragmentation function $f\left(x_{B}^{\text {weak }}\right)$ & 3.0 \\
\hline
\end{tabular}

\section{Appendix B: Simulation of $h^{0} \rightarrow b \bar{b}$ using the POWHEG method}

The NLO differential decay rate in the POWHEG [39] approach is

$$
\begin{aligned}
\mathrm{d} \sigma= & \bar{B}\left(\Phi_{m}\right) \mathrm{d} \Phi_{\mathrm{B}}\left[\Delta_{\mathrm{R}}^{\mathrm{NLO}}\left(p_{T}^{\min }\right)\right. \\
& \left.+\Delta_{\mathrm{R}}^{\mathrm{NLO}}\left(p_{T}^{\min }\right) \frac{R\left(\Phi_{m}, \Phi_{1}\right)}{B\left(\Phi_{m}\right)} \mathrm{d} \Phi_{1}\right],
\end{aligned}
$$

where

$$
\begin{aligned}
\bar{B}\left(\Phi_{m}\right)= & B\left(\Phi_{m}\right)+V\left(\Phi_{m}\right) \\
& +\int\left(R\left(\Phi_{m}, \Phi_{1}\right)-\sum_{i} D_{i}\left(\Phi_{m}, \Phi_{1}\right)\right) \mathrm{d} \Phi_{1} .
\end{aligned}
$$

Here $B\left(\Phi_{m}\right)$ is the leading-order Born differential decay rate, $V\left(\Phi_{m}\right)$ the regularized virtual contribution, $D_{i}\left(\Phi_{m}, \Phi_{1}\right)$ the counter terms regularizing the real emission and $R\left(\Phi_{m}, \Phi_{1}\right)$ the real emission contribution. The leadingorder process has $m$ outgoing partons, with associated phase space $\Phi_{m}$. The virtual and Born contributions depend only on this $m$-body phase space. The real emission phase space, $\Phi_{m+1}$, is factorised into the $m$-body phase space and the phase space, $\Phi_{1}$, describing the radiation of an extra parton.

The Sudakov form factor in the POWHEG method is

$$
\Delta_{R}^{\mathrm{NLO}}=\exp \left[-\int \mathrm{d} \Phi_{1} \frac{R\left(\Phi_{m}, \Phi_{1}\right)}{B\left(\Phi_{m}\right)} \theta\left(k_{T}\left(\Phi_{m}, \Phi_{1}\right)-p_{T}\right)\right]
$$



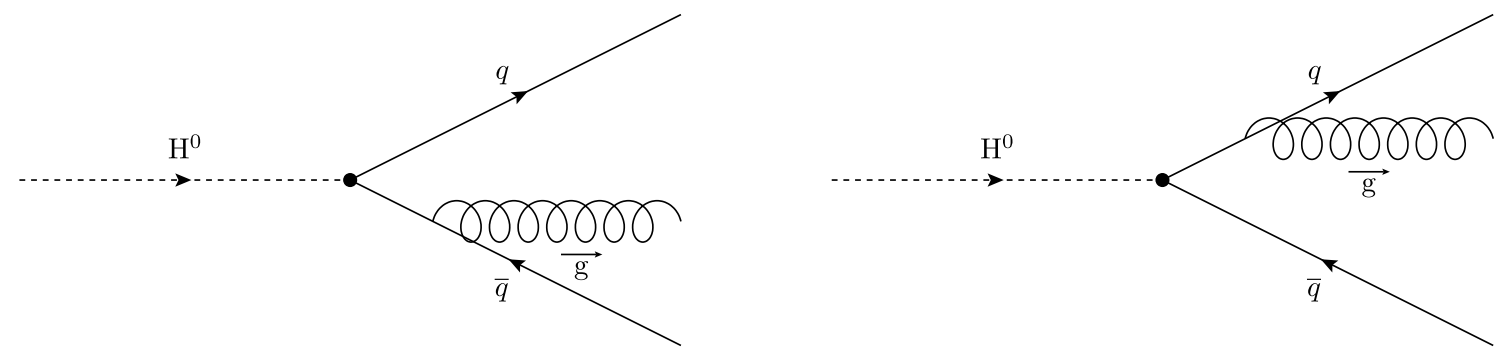

Fig. 9 The two real-emission processes contributing to the NLO decay rate

where $k_{T}\left(\Phi_{m}, \Phi_{1}\right)$ is the transverse momentum of the emitted parton.

In order to implement the decay of the Higgs boson in the POWHEG scheme in Herwig++ we need to generate the Born configuration according to Eq. (5) and the subsequent hardest emission according to Eq. (6). The generation of the truncated and vetoed parton showers from these configurations then proceeds as described in Refs. [29, 52, 54, 55].

The virtual contribution for $h^{0} \rightarrow b \bar{b}$ was calculated in Ref. [56]. The corresponding real emission contribution, see Fig. 9, is

$$
\begin{aligned}
\left|\mathcal{M}_{R}\right|^{2}= & \left|\mathcal{M}_{2}\right|^{2} \frac{\mathrm{C}_{\mathrm{F}} 8 \pi \alpha_{\mathrm{S}}}{M_{H}^{2}\left(1-4 \mu^{2}\right)} \\
& \times\left[2+\frac{1-x_{q}}{1-x_{\bar{q}}}+\frac{\left(8 \mu^{4}-6 \mu^{2}+1\right)}{\left(1-x_{q}\right)\left(1-x_{\bar{q}}\right)}\right. \\
& -2\left(1-4 \mu^{2}\right) \frac{1}{1-x_{q}}-2 \mu^{2}\left(1-4 \mu^{2}\right) \frac{1}{\left(1-x_{q}\right)^{2}} \\
& \left.+\left(x_{q} \leftrightarrow x_{\bar{q}}\right)\right],
\end{aligned}
$$

where $\mathcal{M}_{2}$ is the leading-order matrix element, $\mathrm{C}_{\mathrm{F}}=\frac{4}{3}, m_{q}$ is the mass of the bottom quark, $M_{H}$ is the mass of the Higgs boson, $\mu=\frac{m_{q}}{M_{H}}$ and $x_{i}=\frac{2 E_{i}}{M_{H}}$. We use the Catani-Seymour subtraction scheme [57] where the counter terms are

$$
\begin{aligned}
D_{i}= & \mathrm{C}_{\mathrm{F}} \frac{8 \pi \alpha_{S}}{s}\left|\mathcal{M}_{2}\right|^{2} \frac{1}{1-x_{j}} \\
& \times\left\{\frac{2\left(1-2 \mu^{2}\right)}{2-x_{i}-x_{j}}-\sqrt{\frac{1-4 \mu^{2}}{x_{j}^{2}-4 \mu^{2}}} \frac{x_{j}-2 \mu^{2}}{1-2 \mu^{2}}\right. \\
& \left.\times\left[2+\frac{x_{i}-1}{x_{j}-2 \mu^{2}}+\frac{2 \mu^{2}}{1-x_{j}}\right]\right\},
\end{aligned}
$$

where for $D_{i}, i$ is the emitting parton and $j$ is the spectator parton. In practice, as the counter terms can become negative in some regions, we use

$$
\begin{aligned}
& R\left(\Phi_{m}, \Phi_{1}\right)-\sum_{i} D_{i}\left(\Phi_{m}, \Phi_{1}\right) \\
& \quad=\sum_{i}\left[\frac{R\left(\Phi_{m}, \Phi_{1}\right)\left|D_{i}\left(\Phi_{m}, \Phi_{1}\right)\right|}{\sum_{j}\left|D_{j}\left(\Phi_{m}, \Phi_{1}\right)\right|}-D_{i}\left(\Phi_{m}, \Phi_{1}\right)\right]
\end{aligned}
$$

We have also regulated singularities in the virtual term $V\left(\Phi_{m}\right)$ with the integrated counter terms from the CataniSeymour subtraction scheme allowing us to generate the Born configuration according to $\bar{B}\left(\Phi_{m}\right)$.

The hardest emission for each leg is generated according to

$$
\begin{aligned}
\Delta_{i \mathrm{R}}^{\mathrm{NLO}}= & \exp \left[-\frac{M_{H}^{2}}{16 \pi^{2}\left(1-4 \mu^{2}\right)^{\frac{1}{2}}}\right. \\
& \times \int \mathrm{d} x_{1} \mathrm{~d} x_{2} \mathrm{~d} \phi \frac{R\left(\Phi_{m+1}\right)}{B\left(\Phi_{m}\right)} \frac{\left|D_{i}\right|}{\sum_{j}\left|D_{j}\right|} \\
& \left.\times \theta\left(k_{T}\left(\Phi_{m}, \Phi_{1}\right)-p_{T}\right)\right]
\end{aligned}
$$

However this form is not suitable for the generation of the hardest emission. Instead we perform a Jacobian transformation and use the transverse momentum, $p_{T}$, rapidity, $y$, and azimuthal angle, $\phi$, of the radiated gluon to define the phase space $\Phi_{1}$.

The momenta of the Higgs boson decay products are

$$
\begin{gathered}
p_{1}=\frac{M_{H}}{2}\left(x_{1} ;-x_{\perp} \cos (\phi),-x_{\perp} \sin (\phi),\right. \\
\left. \pm \sqrt{x_{1}^{2}-x_{\perp}^{2}-4 \mu^{2}}\right), \\
p_{2}=\frac{M_{H}}{2}\left(x_{2} ; 0,0,-\sqrt{x_{2}^{2}-4 \mu^{2}}\right), \\
p_{3}=\frac{M_{H}}{2}\left(x_{3} ; x_{\perp} \cos (\phi), x_{\perp} \sin (\phi), \pm \sqrt{x_{3}^{2}-x_{\perp}^{2}}\right),
\end{gathered}
$$

where partons 1,2, 3 are the radiating bottom quark, spectator antibottom quark and radiated gluon, respectively. The energy fractions $x_{i}=\frac{2 E_{i}}{M_{H}}$ and $x_{\perp}=\frac{2 p_{T}}{M_{H}}$. Using the conservation of momentum in the $z$-direction and $x_{1}+x_{2}+x_{3}=2$ 
gives

$x_{\perp}^{2}=\left(2-x_{1}-x_{2}\right)^{2}-\frac{\left(-2+2 x_{1}+2 x_{2}-x_{2} x_{1}-x_{2}^{2}\right)^{2}}{x_{2}^{2}-4 \mu^{2}}$.

Together with the definition, $x_{3}=x_{\perp} \cosh y$, we obtain the Jacobian

$\left|\frac{\partial x_{1} \partial x_{2}}{\partial p_{T} \partial y}\right|=\frac{x_{\perp}}{M_{H}} \frac{x_{\perp}\left(x_{2}^{2}-4 \mu^{2}\right)^{\frac{3}{2}}}{\left(x_{1} x_{2}-2 \mu^{2}\left(x_{1}+x_{2}\right)+x_{2}^{2}-x_{2}\right)}$,

for the transformation of the radiation variables.

We can then generate the additional radiation according to Eq. (10) using the veto algorithm [3]. To achieve this we use an overestimate of the integrand in the Sudakov form factor, $f\left(p_{T}\right)=\frac{c}{p_{T}}$, where $c$ is a suitable constant. We first generate an emission according to

$\Delta_{R}^{\text {over }}\left(p_{T}\right)=\exp \left[-\int_{p_{T}}^{p_{T}^{\max }} \int_{y_{\min }}^{y_{\max }} \mathrm{d} p_{T} \mathrm{~d} y \frac{c}{p_{T}}\right]$,

using this overestimate, where $y_{\max }=\cosh ^{-1}\left(\frac{M_{H}}{2 p_{T}^{\min }}\right), y_{\min }=$ $-y_{\max }, p_{T}^{\max }$ is the maximum possible transverse momentum of the gluon and $p_{T}^{\min }$ is a parameter set in the model, taken to be $1 \mathrm{GeV}$.

The trial value of the transverse momentum is obtained by solving $\mathcal{R}=\Delta_{R}^{\text {over }}$, where $\mathcal{R}$ is a random number in $[0,1]$, i.e.

$p_{T}=p_{T}^{\max } \mathcal{R}^{\frac{1}{c\left(y_{\max }-y_{\min }\right)}}$.

Once the trial $p_{T}$ has been generated, $y$ and $\phi$ are also generated uniformly between $\left[y_{\min }, y_{\max }\right]$ and $[0,2 \pi]$, respectively. The energy fractions of the partons are obtained using the definition $x_{3}=x_{\perp} \cosh y$,

$$
\begin{aligned}
x_{1}= & \frac{1}{2\left(x_{3}-1\right)-\frac{x_{\perp}^{2}}{2}}\left\{3 x_{3}-2+\frac{x_{\perp}^{2}}{2} x_{3}-x_{\perp}^{2}-x_{3}^{2}\right. \\
& \left. \pm \sqrt{\left(x_{3}^{2}-x_{\perp}^{2}\right)\left(\left(x_{3}-1\right)\left(4 \mu^{2}+x_{3}-1\right)-\mu^{2} x_{\perp}^{2}\right)}\right\}
\end{aligned}
$$

and $x_{2}$ using energy conservation. As there are two solutions for $x_{1}$ both solutions must be kept and used to calculate the weight for a particular trial $p_{T}$. The signs of the $z$-components of the momenta are fixed by the sign of the rapidity and momentum conservation. Any momentum configurations outside of the physically allowed phase space are rejected and a new set of variables generated. The momentum configuration is accepted with a probability given by the ratio of the true integrand to the overestimated value. If the configuration is rejected, the procedure continues with $p_{T}^{\max }$ set to the rejected $p_{T}$ until the trial value of $p_{T}$ is accepted or falls below the minimum allowed value, $p_{T}^{\min }$. This procedure generates the radiation variables correctly as shown in Ref. [3].

This procedure is used to generate a trial emission from both the bottom and antibottom. The hardest potential emission is then selected which correctly generates events according to Eq. (10) using this competition algorithm.

\section{References}

1. P.Z. Skands, Tuning Monte Carlo generators: the Perugia tunes. Phys. Rev. D 82, 074018 (2010). arXiv: 1005.3457

2. B. Cooper, J. Katzy, M. Mangano, A. Messina, L. Mijovic et al., Monte Carlo tuning in the presence of matching. arXiv:1109.5295

3. T. Sjöstrand, S. Mrenna, P. Skands, PYTHIA 6.4 Physics and Manual. J. High Energy Phys. 05, 026 (2006). hep-ph/0603175

4. A. Buckley, H. Hoeth, H. Lacker, H. Schulz, J.E. von Seggern, Systematic event generator tuning for the LHC. Eur. Phys. J. C 65, 331-357 (2010). arXiv:0907.2973

5. J.M. Butterworth, A.R. Davison, M. Rubin, G.P. Salam, Jet substructure as a new Higgs search channel at the LHC. Phys. Rev. Lett. 100, 242001 (2008). arXiv:0802.2470

6. S.D. Ellis, C.K. Vermilion, J.R. Walsh, Techniques for improved heavy particle searches with jet substructure. Phys. Rev. D 80, 051501 (2009). arXiv:0903.5081

7. S.D. Ellis, C.K. Vermilion, J.R. Walsh, Recombination algorithms and jet substructure: pruning as a tool for heavy particle searches. Phys. Rev. D 81, 094023 (2010). arXiv:0912.0033

8. G.D. Kribs, A. Martin, T.S. Roy, M. Spannowsky, Discovering the Higgs boson in new physics events using jet substructure. Phys. Rev. D 81, 111501 (2010). arXiv:0912.4731

9. G.D. Kribs, A. Martin, T.S. Roy, M. Spannowsky, Discovering Higgs bosons of the MSSM using jet substructure. Phys. Rev. D 82, 095012 (2010). arXiv:1006.1656

10. G.D. Kribs, A. Martin, T.S. Roy, Higgs discovery through toppartners using jet substructure. arXiv:1012.2866

11. J.M. Butterworth, J.R. Ellis, A.R. Raklev, G.P. Salam, Discovering baryon-number violating neutralino decays at the LHC. Phys. Rev. Lett. 103, 241803 (2009). arXiv:0906.0728

12. CDF Collaboration, T. Aaltonen et al., Study of substructure of high transverse momentum jets produced in proton-antiproton collisions at $\sqrt{s}=1.96 \mathrm{TeV}$. arXiv: 1106.5952

13. Y. Bai, J. Shelton, Composite octet searches with jet substructure. arXiv: 1107.3563

14. C.K. Vermilion, Jet substructure at the Large Hadron Collider: harder, better, faster, stronger. arXiv:1101.1335. PhD thesis

15. T. Plehn, G.P. Salam, M. Spannowsky, Fat jets for a light Higgs. Phys. Rev. Lett. 104, 111801 (2010). arXiv:0910.5472

16. M.H. Seymour, Searches for new particles using cone and cluster jet algorithms: a comparative study. Z. Phys. C 62, 127-138 (1994)

17. A. Abdesselam, E.B. Kuutmann, U. Bitenc, G. Brooijmans, J. Butterworth et al., Boosted objects: a probe of beyond the standard model physics. Eur. Phys. J. C 71, 1661 (2011). arXiv:1012.5412

18. I. Feige, M. Schwartz, I. Stewart, J. Thaler, Precision jet substructure from boosted event shapes. arXiv: 1204.3898

19. A. Altheimer, S. Arora, L. Asquith, G. Brooijmans, J. Butterworth et al., Jet substructure at the Tevatron and LHC: new results, new tools, new benchmarks. J. Phys. G 39, 063001 (2012). arXiv: 1201.0008

20. S. Yang, Q.-S. Yan, Searching for heavy charged Higgs boson with jet substructure at the LHC. J. High Energy Phys. 1202, 074 (2012). arXiv:1111.4530 
21. J.M. Butterworth, B.E. Cox, J.R. Forshaw, WW scattering at the CERN LHC. Phys. Rev. D 65, 096014 (2002). hep-ph/0201098

22. J.M. Butterworth, J.R. Ellis, A.R. Raklev, Reconstructing sparticle mass spectra using hadronic decays. J. High Energy Phys. 05, 033 (2007). hep-ph/0702150

23. Measurement of jet mass and substructure for inclusive jets in $s=$ $7 \mathrm{TeV}$ pp collisions with the atlas experiment. Tech. Rep. ATLASCONF-2011-073, CERN, Geneva, May 2011

24. Atlas sensitivity to the standard model Higgs in the hw and hz channels at high transverse momenta. Tech. Rep. ATL-PHYSPUB-2009-088, CERN, Geneva, August 2009

25. Study of jet substructure in pp collisions at $7 \mathrm{TeV}$ in $\mathrm{cms}$. Tech. Rep. CMS PAS JME 10-013, CERN, Geneva, March 2011

26. G. Corcella et al., HERWIG 6: an event generator for hadron emission reactions with interfering gluons (including supersymmetric processes). J. High Energy Phys. 01, 010 (2001). hep-ph/0011363

27. G. Corcella et al., HERWIG 6.5 release note. hep-ph/0210213

28. J.M. Butterworth, J.R. Forshaw, M.H. Seymour, Multi-parton interactions in photoproduction at HERA. Z. Phys. C 72, 637-646 (1996). hep-ph/9601371

29. M. Bähr et al., Herwig++ physics and manual. Eur. Phys. J. C 58, 639-707 (2008). arXiv:0803.0883

30. K. Arnold, L. d'Errico, S. Gieseke, D. Grellscheid, K. Hamilton et al., Herwig++ 2.6 release note. arXiv: 1205.4902

31. G. Marchesini, B.R. Webber, Simulation of QCD coherence in heavy quark production and decay. Nucl. Phys. B 330, 261 (1990)

32. S. Gieseke, P. Stephens, B. Webber, New formalism for QCD parton showers. J. High Energy Phys. 12, 045 (2003). hep-ph/0310083

33. M. Bähr, S. Gieseke, M.H. Seymour, Simulation of multiple partonic interactions in Herwig++. arXiv:0803.3633

34. M. Bähr, J.M. Butterworth, M.H. Seymour, The underlying event and the total cross section from Tevatron to the LHC. arXiv: 0806.2949

35. S. Gieseke, C. Röhr, A. Siodmok, Colour reconnections in Herwig++. arXiv:1206.0041

36. S. Gieseke, C. Röhr, A. Siodmok, Multiple partonic interaction developments in Herwig++. arXiv: 1110.2675

37. G. Aad et al. (ATLAS Collaboration), Jet mass and substructure of inclusive jets in $\sqrt{s}=7 \mathrm{TeV}$ pp collisions with the ATLAS experiment. J. High Energy Phys. 1205, 128 (2012). arXiv:1203.4606

38. G. Aad et al. (ATLAS Collaboration), ATLAS measurements of the properties of jets for boosted particle searches. arXiv: 1206.5369

39. P. Nason, A new method for combining NLO QCD with shower Monte Carlo algorithms. J. High Energy Phys. 11, 040 (2004). hep-ph/0409146

40. S. Frixione, P. Nason, C. Oleari, Matching NLO QCD computations with parton shower simulations: the POWHEG method. J. High Energy Phys. 11, 070 (2007). arXiv:0709.2092

41. B.R. Webber, A QCD model for jet fragmentation including soft gluon interference. Nucl. Phys. B 238, 492 (1984)

42. D. Amati, G. Veneziano, Preconfinement as a property of perturbative QCD. Phys. Lett. B 83, 87 (1979)
43. A. Buckley et al., General-purpose event generators for LHC physics. arXiv: 1101.2599

44. P. Abreu et al. (DELPHI Collaboration), Tuning and test of fragmentation models based on identified particles and precision event shape data. Z. Phys. C 73, 11-60 (1996)

45. J. Pumplin, D. Stump, R. Brock, D. Casey, J. Huston et al., Uncertainties of predictions from parton distribution functions, 2: the Hessian method. Phys. Rev. D 65, 014013 (2001). hep-ph/0101032

46. J. Pumplin, D. Stump, W. Tung, Multivariate fitting and the error matrix in global analysis of data. Phys. Rev. D 65, 014011 (2001). hep-ph/0008191

47. Y.L. Dokshitzer, G.D. Leder, S. Moretti, B.R. Webber, Better jet clustering algorithms. J. High Energy Phys. 08, 001 (1997). hep-ph/9707323

48. M. Wobisch, Measurement and QCD analysis of jet cross sections in deep-inelastic positron proton collisions at $s^{* *}(1 / 2)=$ $300 \mathrm{GeV}$. DESY-THESIS-2000-049

49. M. Cacciari, G.P. Salam, Dispelling the $N^{3}$ myth for the $k_{t}$ jetfinder. Phys. Lett. B 641, 57-61 (2006). hep-ph/0512210

50. K. Hamilton, P. Richardson, A simulation of QCD radiation in top Quark Decays. J. High Energy Phys. 02, 069 (2007). hep-ph/0612236

51. K. Hamilton, A positive-weight next-to-leading order simulation of weak boson pair production. J. High Energy Phys. 1101, 009 (2011). arXiv:1009.5391

52. K. Hamilton, P. Richardson, J. Tully, A positive-weight next-toleading order Monte Carlo simulation for Higgs boson production. J. High Energy Phys. 04, 116 (2009). arXiv:0903.4345

53. R. Barate et al. (ALEPH Collaboration), Studies of quantum chromodynamics with the ALEPH detector. Phys. Rep. 294, 1-165 (1998)

54. K. Hamilton, P. Richardson, J. Tully, A positive-weight next-toleading order Monte Carlo simulation of Drell-Yan vector boson production. arXiv:0806.0290

55. L. D'Errico, P. Richardson, A positive-weight next-to-leadingorder Monte Carlo simulation of deep inelastic scattering and Higgs boson production via vector boson fusion in Herwig++. arXiv: 1106.2983

56. E. Braaten, J.P. Leveille, Higgs boson decay and the running mass. Phys. Rev. D 22, 715 (1980)

57. S. Catani, S. Dittmaier, M.H. Seymour, Z. Trócsányi, The dipole formalism for next-to-leading order QCD calculations with massive partons. Nucl. Phys. B 627, 189-265 (2002). hep-ph/0201036

58. A. Buckley, J. Butterworth, L. Lonnblad, H. Hoeth, J. Monk, et al., Rivet user manual. arXiv:1003.0694

59. C. Amsler, et al. (Particle Data Group), Review of particle physics. Phys. Lett. B 667, 1-1340 (2008). doi:10.1016/j.physletb.2008. 07.018

60. G. Barker, E. Ben-Haim, M. Feindt, U. Kerzel, P. Roudeau, L. Ramler, A. Savoy-Navarro, A study of the b-quark fragmentation function with the DELPHI detector at LEP I. DELPHI-2002069-CONF-603, CERN, Geneva, July 2002 